\title{
Abdominal Aortic Aneurysm Screening: How Many Life Years Lost from Underuse of the Medicare Screening Benefit?
}

\author{
N. Olchanski, MS, A. Winn, MPP, J. T. Cohen, PhD, and P. J. Neumann, ScD \\ Center for the Evaluation of Value and Risk in Health, Institute for Clinical Research and Health Policy Studies, Tufts Medical Center, Boston, \\ MA, USA.
}

BACKGROUND: Since 2007, Medicare has provided one-time abdominal aortic aneurysm (AAA) screening for men with smoking history, and men and women with a family history of AAA as part of its Welcome to Medicare visit.

OBJECTIVE: We examined utilization of the new AAA screening benefit and estimated how increased utilization could influence population health as measured by life years gained. Additionally, we explored the impact of expanding screening to women with smoking history.

DESIGN: Analysis of Medicare claims and a simulation model to estimate the effects of screening, using published data for parameter estimates.

SETTING: AAA screening in the primary care setting. PATIENTS: Newly-enrolled Medicare beneficiaries aged 65 years, with smoking history or family history of AAA. MAIN MEASURES: Life expectancy, 10-year survival rates.

KEY RESULTS: Medicare data revealed low utilization of AAA screening, under $1 \%$ among those eligible. We estimate that screening could increase life expectancy per individual invited to screening for men with smoking history (0.11 years), with family history of AAA (0.17 years), and women with family history (0.08 years), and smoking history (0.09 years). Average gains of 131 life years per 1,000 persons screened for AAA compare favorably with the grade B United States Preventive Services Task Force (USPSTF) recommendation for breast cancer screening, which yields 95-128 life years per 1,000 women screened. These findings were robust over a range of scenarios.

LIMITATIONS: The simulation results reflect assumptions regarding AAA prevalence, treatment, and outcomes in specific populations based on published research and US survey data. Published data on women were limited.

CONCLUSIONS: The Welcome to Medicare and AAA screening benefits have been underutilized. Increasing utilization of AAA screening would yield substantial gains in life expectancy. Expanding screening to women

Electronic supplementary material The online version of this article (doi:10.1007/s11606-014-2831-z) contains supplementary material, which is available to authorized users.

Received June 21, 2013

Revised November 25, 2013

Accepted March 2, 2014

Published online April 9, 2014 with smoking history also has the potential for substantial health benefits.

KEY WORDS: abdominal aortic aneurysm; screening; medicare; health policy; health services research.

J Gen Intern Med 29(8):1155-61

DOI: $10.1007 / \mathrm{s} 11606-014-2831-\mathrm{Z}$

(C) Society of General Internal Medicine 2014

\section{INTRODUCTION}

Abdominal aortic aneurysm (AAA) is a condition that may exist and worsen for years to decades without symptoms, but over time may lead to a rupture of the aorta, with a high rate of fatality. A person with known AAA may be monitored by a physician and offered surgical intervention to lower the risk of rupture when risk of death from rupture exceeds the risks from surgical treatment. Because the condition is generally asymptomatic, such persons are often identified through AAA screening or via incidental abdominal imaging findings.

Since 1 January 2007, provisions of the SAAAVE (Screening Abdominal Aortic Aneurysm Very Efficiently) Act in the US have provided a free, one-time, ultrasound AAA screening for qualified Medicare beneficiaries as part of their Welcome to Medicare examination within the first 12 months of enrollment. Men who have smoked at least 100 cigarettes during their life, and men and women with a family history of AAA qualify for the benefit. Long-term follow-up results from the most recent and largest study of AAA screening in men, the Multicenter Aneurysm Screening Study (MASS), found that a one-time screening program for men is very cost-effective $(7,600$ pounds, or roughly $\$ 12,000$, per quality adjusted life year gained at the 10-year mark). ${ }^{1}$ Other studies have also reported favorable cost-effectiveness for the screening of men for AAA. ${ }^{2-5}$

Despite the availability of the AAA screening benefit and its cost-effectiveness, recent reports indicate very low uptake. ${ }^{6}$ One study of Medicare claims data from 2004 to 2008 found that fewer than $10 \%$ of eligible men aged 65 years received any abdominal ultrasonography after $2007 .^{7}$ 
The authors did not report the proportion of Medicare beneficiaries who had taken advantage of the screening benefit. Our study aims to 1) examine the adoption of AAA screening and the impact of the 2007 SAAAVE Act, and 2) estimate the impact on years of life potentially saved by different levels of individual participation in AAA screening in the US.

A second issue for AAA screening is whether the current Medicare benefit is optimally targeted. As noted, the Medicare screening is available to men but not women who have smoked at least 100 cigarettes during their lifetimes. Evidence for AAA screening in women is limited because most of the relevant studies and recommendations have focused on men. ${ }^{8}$ Meta-analysis of randomized controlled studies has found that screening older men for AAA reduces AAA-related mortality, but fails to reduce all-cause mortality significantly. Only the Chichester trial in the UK examined the impact of screening women, and that relatively small study did not find a significant difference in AAA-related or all cause mortality at 5 years. ${ }^{9}$ To date, women have been excluded from the large trials of screening for AAA. ${ }^{10}$

Nonetheless, screening women with risk factors may make sense. Although the prevalence of AAA is higher in men, the rate of rupture and prognosis for survival is worse in women. ${ }^{10-13}$ Lederle reports that "although AAAs are four to six times more common in men than in women, more than one-third of all AAA deaths occur in women." ${ }^{.4}$ Thus, early identification of AAA in a woman may yield greater benefits. The one study that evaluated the costeffectiveness of screening women found that it is costeffective $(\$ 5,900$ per life year gained). The authors concluded that the low prevalence of AAA is balanced by higher mortality rates associated with aneurysm ruptures in women. ${ }^{15}$ Our paper explores the impact of screening women with smoking history, a group that is not currently eligible for the Medicare screening benefit.

\section{METHODS}

Assessing the extent to which AAA screening has been adopted requires estimating the size of the eligible population and utilization of the AAA screening benefit. We analyzed Medicare claims data to estimate utilization of the Welcome to Medicare examination (G-codes G0344, used prior to 2009, and G0402) among new enrollees and utilization of the new AAA screening benefit (G-code G0389).

We used Medicare standard analytic files covering $5 \%$ of the population for the years 2005-2009 to estimate benefit utilization during the 2 years prior to and following the adoption of the 2007 SAAAVE Act. The study was based on deidentified secondary data and received institutional review board (IRB) exempt status. Eligible new enrollees included new beneficiaries, age 64 or 65 years, in the
Master Summary File. Because the claims data dates of service are masked, we assumed that screening that occurred in the same or following quarter, as the initial Welcome to Medicare visit represented instances of beneficiaries using the Medicare screening benefit.

To estimate the size of the population of 65 -year-olds with a smoking history, we used the National Health Interview Survey (NHIS). To estimate the proportion of individuals with a family history of AAA, we used the Aneurysm Detection and Management (ADAM) study, a large clinical study of AAA management performed in 15 Department of Veterans Affairs medical centers. ${ }^{16}$

To provide additional context, we estimated the number of newly enrolled beneficiaries with AAA diagnosis (ICD-9 codes 441.4 and 441.3). Further, we estimated the size of the population with newly diagnosed AAA as the number of individuals who now had AAA, but who had no claims with AAA diagnosis codes during the year prior to their first diagnosis.

Our second aim was to estimate the potential impact of increasing AAA screening rates among Medicare beneficiaries. We developed a simulation model to project the natural history of AAA, and to estimate how different AAA screening rates among eligible Medicare beneficiaries would influence outcomes. Model assumptions for the base case and sensitivity analyses were based largely on published estimates (Table 1).

To assess the impact of the SAAAVE Act screening benefit, we modeled a 1-year cohort of eligible beneficiaries. The simulated cohort included men aged 65 years with smoking history or family history of AAA, and women aged 65 years with family history of AAA. AAA prevalence for the male population with smoking history was based on a United States Preventive Services Task Force (USPSTF) systematic review of AAA clinical trials. ${ }^{8}$ Since we found no contemporary published estimates of AAA prevalence for men or women with a family history of this condition, we estimated these rates from ADAM study results reported a decade ago by Lederle et al. ${ }^{16,22}$

Figure 1 and Table 1 illustrate the model's structure and assumptions. For the simulated cohort, the base case screening rate was $80 \%$, with some cases of AAA discovered incidentally. These assumptions were based on findings reported by the Multicenter Aneurysm Screening Study (MASS), ${ }^{17}$ a randomized clinical trial of AAA screening from the UK. Because there were no reported estimates for the rate of incidental discovery of AAA, we assumed that $6.6 \%$ of all latent AAAs are discovered incidentally each year, per Lee et al. (equivalent to a 10year discovery rate of $50 \%) .{ }^{18}$ Depending on the size of the aneurysm, clinical guidelines direct patients to either repair surgery or watchful waiting. The aneurysm size distribution was taken from the MASS study, described above. ${ }^{17}$ As the MASS study did not include women, and there are no published estimates of aneurysm size distribution in 
Table 1. Model Input Parameters and Assumptions

\begin{tabular}{|c|c|c|c|}
\hline Assumption & Base Case & Distribution & Source \\
\hline Response Rate to Screening Invitation & $80 \%$ & Beta $(50,556,12,466)$ & 17 \\
\hline Annual Incidental Detection & $6.6 \%$ & Beta $(10,707,151,515)$ & 18 \\
\hline \multicolumn{4}{|l|}{ AAA Prevalence } \\
\hline Male with Smoking History & $6.4 \%$ & Beta $(10,683,156,249)$ & 8 \\
\hline Male with Family History & $8.4 \%$ & Beta $(10,515,204,081)$ & 16 \\
\hline Female with Family History & $2.6 \%$ & Beta $(11,013,108,695)$ & 16 \\
\hline Female with Smoking History & $3.8 \%$ & Beta $(10,395,263,158)$ & 16 \\
\hline \multicolumn{4}{|l|}{ AAA Size } \\
\hline Small $(3-4.4 \mathrm{~cm})$ & $71 \%$ & Dirichlet $(295,90,46)$ & 17 \\
\hline Medium $(4.5-5.4 \mathrm{~cm})$ & $17 \%$ & & 17 \\
\hline Large $(\geq 5.5 \mathrm{~cm})$ & $12 \%$ & & 17 \\
\hline \multicolumn{4}{|l|}{ Annual Transition Probabilities } \\
\hline Small to Medium & $9.3 \%$ & Beta $(11,025,107,527)$ & 5 \\
\hline Medium to Large & $29.5 \%$ & Beta $(14,184,33,898)$ & 5 \\
\hline \multicolumn{4}{|l|}{ Annual Rupture Rates } \\
\hline Small, Men & $0.038 \%$ & Beta $(10,038,2,631,579)$ & 5,13 \\
\hline Small, Women & $0.14 \%$ & Beta $(10,014,6,998,880)$ & 5,13 \\
\hline Medium, Men & $1 \%$ & Beta $(10,101,1,000,000)$ & 10 \\
\hline Medium, Women & $4 \%$ & Beta $(10,417,250,000)$ & 10 \\
\hline Large, Men & $12 \%$ & Beta $(11,364,83,333)$ & 10 \\
\hline Large, Women & $18 \%$ & Beta $(12,195,55,556)$ & 10 \\
\hline Patients unfit or refusing surgery & $18.6 \%$ & Beta $(12,285,53,763)$ & 4,19 \\
\hline \multicolumn{4}{|l|}{ AAA Specific Mortality ( 30 day) } \\
\hline Ruptured Pre-hospital mortality & $59.9 \%$ & Beta $(24,938,16,694)$ & 4 \\
\hline Emergency surgery mortality, Men & $35.6 \%$ & Beta $(15,528,28,090)$ & 12 \\
\hline Emergency surgery mortality, Women & $43 \%$ & Beta $(17,544,23,256)$ & 12 \\
\hline Planned Surgery mortality, Men & $2.6 \%$ & Beta $(10,267,384,615)$ & 12 \\
\hline Planned Surgery mortality, Women & $4.8 \%$ & Beta $(10,469,223,214)$ & 12 \\
\hline \multicolumn{3}{|c|}{ Annual Background Mortality, adjusted by age, gender and smoking status } & National Vital Statistics ${ }^{20,21}$ \\
\hline
\end{tabular}

women, we used the same distribution for both genders. Multiple studies indicate that women with AAA are less likely to be referred for surgery, and that because they more frequently die immediately, women with ruptured AAA are less likely to be admitted to the hospital. ${ }^{10}$ However, it is unclear what characteristics predict the likelihood of surgery, and most data reflect the treatment of men. Therefore, we conservatively assumed equal rates of planned surgery for intact AAA and emergency surgery for ruptured AAA in men and women.

For any aneurysm, detected or missed, there is a chance of rupture, subsequent emergency repair, and possible death. Rates for these outcomes were also based on estimates from the MASS study. ${ }^{5}$ Aneurysm rupture rates were higher in women than in men, as summarized in a meta-analysis and a systematic review of the clinical literature. ${ }^{10,13}$ Aneurysm growth rates over time were based on estimates by Kim at al. from the MASS study and Chichester trial, a randomized multicenter study of AAA screening in the UK. ${ }^{5}$

We estimated AAA-related mortality for death due to ruptured AAA without admission to the hospital, death due to ruptured AAA after an emergency repair procedure, and postoperative mortality after planned repair of intact AAA. We estimated the first category from published estimates from several decision analytic models ${ }^{4}$ based on data from male patients. Because we could identify no data on the proportion of women with ruptured AAA reaching the hospital alive, we used the same estimate for men and women. Gender specific estimates of postoperative mortality after emergency and planned repair procedures were obtained from a large retrospective analysis of the Nationwide Inpatient Sample 2001-2004. ${ }^{12}$

The model accounted for general mortality from other causes, with assumptions based on National Vital Statistics for all causes except AAA. Rates were adjusted for smoking status, with smoking rates taken from the NHIS and the effects of smoking on longevity from published data. ${ }^{20,21}$ All mortality estimates were age, gender, and smoking status specific.

We simulated the impact of screening women with history of smoking, a population at increased risk of AAA, but ineligible for the Medicare screening benefit. Because specific AAA prevalence rates for this population have not been reported in the literature, we estimated prevalence using odds ratios in women reported by the ADAM study. ${ }^{16}$

We performed univariate and multivariate probabilistic sensitivity analyses to identify the most influential assumptions. The probabilistic sensitivity analysis sampled inputs 250 times from specified distributions (Table 1), and generated 1,000 simulated individuals for each set. Finally, we produced $95 \%$ confidence intervals for model outcomes to characterize the effects of parameter uncertainty and the robustness of our findings. Analyses were performed using SAS version 9.3 and Microsoft Excel 2007.

\section{RESULTS}

Our analysis of Medicare data showed uptake of AAA screening of less than $1 \%$ each year among newly enrolled 


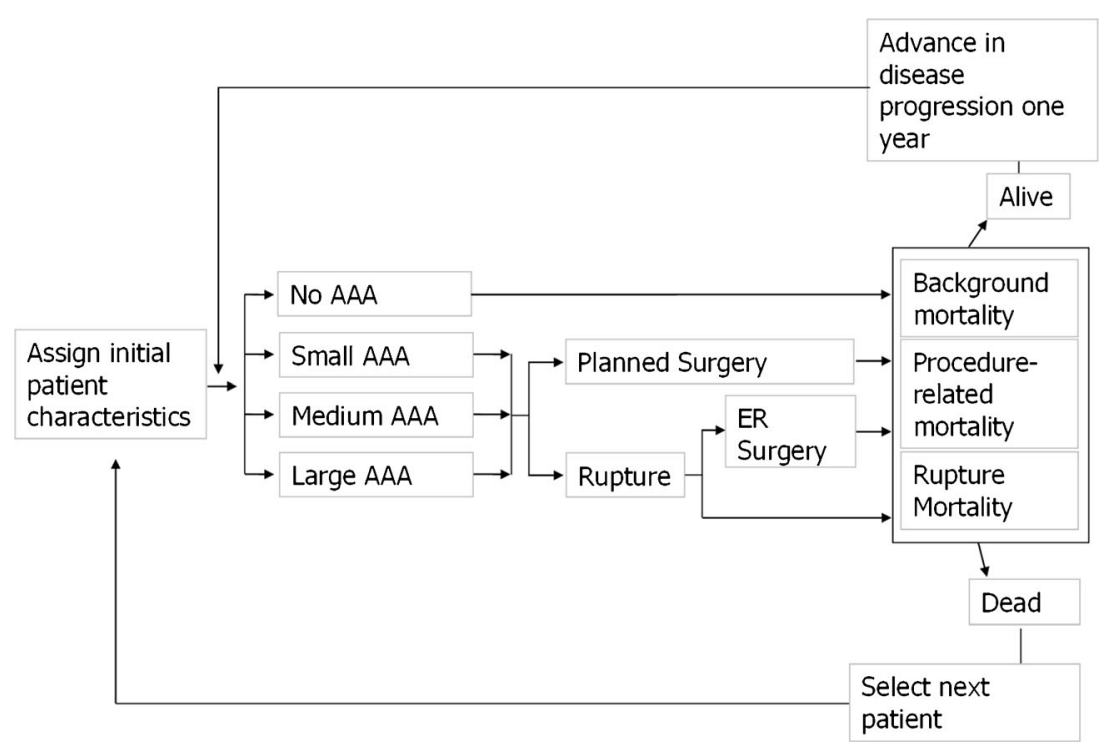

Figure 1. Simulation model structure (annual iteration per simulated individual).

Medicare beneficiaries and those eligible for the screening benefit (Table 2). The number of recently enrolled beneficiaries newly diagnosed with AAA, including ruptured aneurysms, ranged from 6,660 to 9,260 per year, with no decrease from 2006-2009.

Table 3 summarizes the benefits of screening, showing that it increases life expectancy by $0.11-0.17$ years for men and 0.08 years for women. Average life expectancy gains would be even greater if screening rates exceeded our assumed base case value of $80 \%$. Life expectancy gains for men with detected AAA averaged 1.79-1.98 years, with 10year survival increasing by 9.7-10.5\%. Life expectancy gains in women with family history and detected AAA were 2.95 life years, with 10-year survival increasing by $14.5 \%$.

Extrapolating screening benefits from 2007-2012 through 2025 yields over 400,000 saved life years
(Fig. 2). Figure 2 also shows that an additional 291,000 life years can be saved by 2025 if screening rates increase from current levels to $80 \%$ between 2013 and 2018. These gains translate to 131 life years per 1,000 persons screened.

We also examined the potential impact of expanding the Medicare screening benefit to women with smoking history (Table 3). Screening recommendations typically omit this group, but our analysis indicates potential gains of 2.38 life years for individuals with detected AAA, or 0.09 life years on average over all individuals invited to screening. For individuals with AAA, 10-year survival would increase $13.3 \%$.

Our results depend on the assumed AAA prevalence, proportion of eligible individuals screened, AAA rupture rates, and AAA-related mortality rates. Due to space limitations, we detail one-way sensitivity analyses in an

Table 2. Welcome to Medicare Visit and AAA Screening Rates in Newly Enrolled Medicare Beneficiaries in the Years $2005-2009$

\begin{tabular}{|c|c|c|c|c|c|}
\hline & \multicolumn{5}{|c|}{ Year Initially Enrolled in Medicare } \\
\hline & 2005 & 2006 & 2007 & 2008 & 2009 \\
\hline Total new beneficiaries $(\mathrm{N})$ & $2,232,792$ & $2,909,980$ & $3,133,340$ & $3,232,020$ & $3,222,500$ \\
\hline AAA Diagnosis & 21,482 & 22,139 & 22,434 & 22,575 & 22,177 \\
\hline New AAA Diagnosis & & 6,660 & 7,260 & 9,260 & 7,280 \\
\hline \multicolumn{6}{|l|}{ Welcome to Medicare* } \\
\hline All Beneficiaries & 2,176 & 83,860 & 91,300 & 109,000 & 102,400 \\
\hline AAA Screening $^{\dagger}$ & 0 & 0 & 600 & 1,700 & 1,880 \\
\hline Eligible for AAA Screening benefit & 863,298 & 878,326 & 912,112 & $1,022,243$ & 904,081 \\
\hline
\end{tabular}

AAA diagnosis defined by ICD-9 codes 441.4 and 441.3; individuals with new AAA diagnosis had no instances of AAA diagnosis in the year prior to the first diagnosis

"A Welcome to Medicare (WTM) visit in the same or next year as enrolled in Medicare

${ }^{\prime}$ In the same or following quarter as WTM visit

${ }^{*}$ Based on data from NHIS 2005-2010, weighted up to national estimates, with prevalence of AAA in people with family history based on Lederle 2001

Note: National estimates based on 5 \% sample Standard Analytic File. Welcome to Medicare defined by G-codes G0344 and G0402; AAA screening defined by G-code G0389 
Table 3. Simulation Model Results: the Impact of AAA Screening (Average Life Expectancy and 10-Year Survival by Cohort)

\begin{tabular}{|c|c|c|c|c|c|c|c|c|}
\hline & \multicolumn{4}{|c|}{ Average Life Expectancy } & \multicolumn{4}{|c|}{ Prob. Of 10-Year Survival } \\
\hline & $\begin{array}{l}\text { No Screening } \\
\text { Strategy }\end{array}$ & $\begin{array}{l}\text { Screening } \\
\text { Strategy }\end{array}$ & Diff. & $P$ value & $\begin{array}{l}\text { No Screening } \\
\text { Strategy }\end{array}$ & $\begin{array}{l}\text { Screening } \\
\text { Strategy }\end{array}$ & Diff. & $P$ value \\
\hline \multicolumn{9}{|l|}{ Male, Smoker } \\
\hline All invited to screening & 80.49 & 80.60 & 0.11 & $<0.0001$ & $66.0 \%$ & $66.7 \%$ & $0.7 \%$ & $<0.0001$ \\
\hline Diagnosed with AAA & 78.07 & 79.86 & 1.79 & $<0.0001$ & $52.9 \%$ & $62.6 \%$ & $9.7 \%$ & $<0.0001$ \\
\hline \multicolumn{9}{|l|}{ Male, Family History } \\
\hline All invited to screening & 81.68 & 81.84 & 0.17 & $<0.0001$ & $71.1 \%$ & $72.0 \%$ & $0.9 \%$ & $<0.0001$ \\
\hline Diagnosed with AAA & 79.08 & 81.06 & 1.98 & $<0.0001$ & $57.5 \%$ & $68.0 \%$ & $10.5 \%$ & $<0.0001$ \\
\hline \multicolumn{9}{|l|}{ Female, Family History } \\
\hline All invited to screening & 84.14 & 84.22 & 0.08 & $<0.0001$ & $80.1 \%$ & $80.5 \%$ & $0.4 \%$ & $<0.0001$ \\
\hline Diagnosed with AAA & 79.40 & 82.35 & 2.95 & $<0.0001$ & $56.5 \%$ & $71.0 \%$ & $14.5 \%$ & $<0.0001$ \\
\hline \multicolumn{9}{|l|}{ Female, Smoking History } \\
\hline All invited to screening & 81.64 & 81.73 & 0.09 & $<0.0001$ & $72.6 \%$ & $73.1 \%$ & $0.5 \%$ & $<0.0001$ \\
\hline Diagnosed with AAA & 77.79 & 80.17 & 2.38 & $<0.0001$ & $51.2 \%$ & $64.4 \%$ & $13.3 \%$ & $<0.0001$ \\
\hline
\end{tabular}

online appendix. In summary, the life year gain per person remained positive for all tested assumptions. Multivariate sensitivity analysis results (Table 4) show that screening benefits for all four modeled populations persist across all scenarios. None of the $95 \%$ confidence intervals for life expectancy gain per person include zero, indicating that in all scenarios, screening improves life expectancy relative to no screening.

\section{DISCUSSION}

Analysis of Medicare data showed that preventive services, such as the Welcome to Medicare (WTM) visit for new enrollees, and especially the AAA screening benefit established by the SAAAVE Act of 2007, have been underutilized. Underutilization of the AAA screening benefit likely reflects underutilization of the WTM visit, which provides a range of screening services. The Centers for Medicare and Medicaid Services (CMS) reports that from 2005 to 2009 , only $10 \%$ of the nearly one million eligible enrollees each year took advantage of the WTM benefit. ${ }^{6}$ Reasons for the low adoption rate included the limited time period during which the WTM benefit must be used (within 12 months of enrollment), and a patient out-ofpocket deductible (\$124 for 2006). ${ }^{23}$ Additionally, physician reimbursement for the WTM visit had been slightly lower than an initial wellness visit. ${ }^{6}$

Recent changes may increase preventive service utilization. Beginning in 2009, the WTM visit eligibility period was expanded. In 2011, the Patient Protection and Affordable Care Act (ACA) instituted an Annual Wellness Visit, available to beneficiaries for free. According to CMS, "over 780,000 beneficiaries received an Annual Wellness Visit between January 1 and June 10 [2011]." ${ }^{24}$ While it is too early to tell whether AAA screening rates will increase in lockstep with the utilization of other preventive services, AAA screening rates should be monitored as new data become available.

Our simulation showed that increasing the utilization of AAA screening to $80 \%$ of the eligible population would

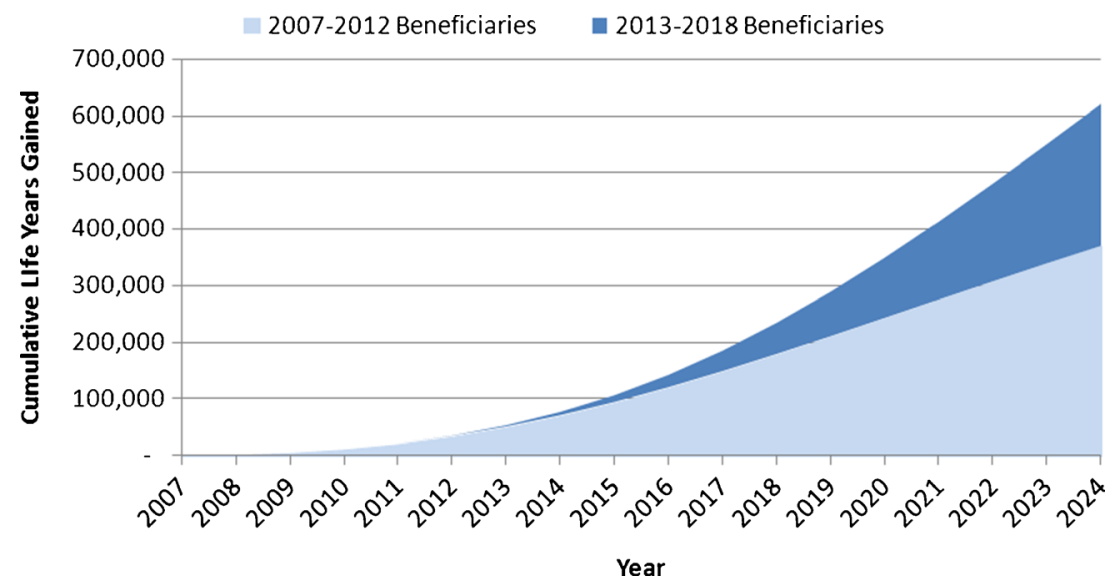

Figure 2. Cumulative life years gained from the use of Medicare AAA screening benefit, compared with current use levels. 
Table 4. Multivariate Sensitivity Analysis Results

\begin{tabular}{|c|c|c|c|c|}
\hline & No Screening Strategy & Screening Strategy & Diff. & $95 \% \mathrm{CI}$ \\
\hline \multicolumn{5}{|l|}{ Male, Smoking History } \\
\hline All invited to screening & 80.48 & 80.60 & 0.11 & $(0.07,0.16)$ \\
\hline Diagnosed with AAA & 78.18 & 80.00 & 1.82 & $(1.62,2.01)$ \\
\hline \multicolumn{5}{|l|}{ Male, Family History } \\
\hline All invited to screening & 81.66 & 81.84 & 0.17 & $(0.13,0.22)$ \\
\hline Diagnosed with AAA & 79.01 & 81.08 & 2.08 & $(1.91,2.24)$ \\
\hline \multicolumn{5}{|l|}{ Female, Family History } \\
\hline All invited to screening & 84.19 & 84.24 & 0.06 & $(0.01,0.10)$ \\
\hline Diagnosed with AAA & 80.20 & 82.36 & 2.16 & $(1.86,2.47)$ \\
\hline \multicolumn{5}{|l|}{ Female, Smoking History } \\
\hline All invited to screening & 81.63 & 81.71 & 0.08 & $(0.03,0.13)$ \\
\hline Diagnosed with AAA & 78.29 & 80.36 & 2.07 & $(1.83,2.32)$ \\
\hline
\end{tabular}

*Probabilistic sensitivity analysis sampled inputs 250 times from specified distributions (Table 1), and generated 1,000 simulated individuals for each set

substantially increase life expectancy. To put the findings into perspective, gaining 131 life years per 1,000 persons screened for AAA is comparable to the gains from the grade $B$ USPSTF recommendation for breast cancer screening (biennial screening mammography for women aged 50 to 74 years), which would yield 95-128 life years per 1,000 women screened. ${ }^{25}$ Our findings were robust over a range of scenarios and sensitivity analyses. While it may be optimistic to assume $80 \%$ utilization for screening, similar rates were observed in practice in the MASS clinical trial. ${ }^{17}$ Moreover, our sensitivity analysis evaluated the impact of alternative screening rate assumptions.

For women with risk factors, our analysis shows that AAA screening yields larger life expectancy gains than the screening of men already covered by Medicare. This larger gain may in part reflect the fact that, setting aside the risk posed by AAA, women have longer life expectancies and hence represent a greater opportunity for life year gains. It may also reflect the higher mortality rates from AAA events in women. Aggregate gains for this high-risk group would be 400,000 life years, exceeding by more than an order of magnitude the aggregate gains for low-risk groups (no family history and no history of smoking) of men $(36,000$ life years) and women (21,000 life years). Clinical and economic studies of screening have perhaps focused on males because of their higher AAA prevalence. Higher prevalence among men, however, does not necessarily imply greater benefits, because other factors, such as mortality risk per event and life years gained per averted fatality, must be appropriately taken into account.

Our analysis has several limitations. First, improper coding may have caused us to underestimate current screening rates and the utilization of WTM visits by new enrollees. However, providers familiar with the new benefits most likely used the correct codes.

Second, the simulation results reflect assumptions regarding AAA prevalence in specific populations, outcomes of different AAA management strategies, mortality, and detection levels associated with screening. These estimates were developed from published reports of clinical trials, US survey data, and other sources. Both natural history and treatment patterns are less well-studied in women, and screening for women has not been studied in large cohorts. Therefore, we estimated the prevalence for this group indirectly. Further, because of gaps in published epidemiology and outcome data for women with AAA, we assumed in some cases that treatment rates and AAA size distributions for women are similar to the corresponding distributions for men. Finally, there may be some selection bias if individuals who seek screening do not have the same risk for aneurysm as the eligible population. We tested the robustness of our findings by conducting both univariate and multivariate sensitivity analyses.

Preventive care is frequently identified as a means to improve health and reduce costs. Although previous research has found that preventive services often do not save money, ${ }^{26,27}$ AAA screening does provide an opportunity to save lives at a modest cost. ${ }^{1-5,15,18}$ Our results require action from policy makers to increase utilization of AAA screening and to ensure its availability to populations for which it represents good value. To ensure the best value from screening, it is important to target those populations most at risk. Recent AAA predictive modeling research indicates that screening based on risk scores that incorporate factors beyond just family history could be even more effective than criteria used previously. ${ }^{28}$

Recent policy changes have helped to make preventive services more accessible to Medicare beneficiaries by eliminating out-of-pocket costs and extending the eligibility time frames for wellness visits. However, further steps are needed to both raise awareness of the benefits of AAA screening and to eliminate factors discouraging its use. Policymakers should review patient and physician financial incentives, and how AAA screening fits into the flow of patient-provider interactions. De-linking the benefit from the Welcome to Medicare visit, while retaining it as a onetime screen, could increase adoption by providing more flexibility and time for beneficiaries to take advantage of it. Additionally, there are no vocal patient advocacy groups for AAA and there is limited awareness among the US 
population. AAA screening rates might increase if awareness of this condition were raised by direct-to-consumer communications. Finally, our findings suggest that policymakers should consider extending the AAA screening benefit to women with smoking history.

Acknowledgements: The authors wish to acknowledge the contributions of Dr. Robert Zwolak in reviewing the draft manuscript and in providing comments from a clinical and policy perspective.

Financial Support: This research was supported by Medtronic, Inc., Minneapolis, $M N$

Prior Presentations: The findings from this study were presented at the annual meeting of the International Society for Pharmacoeconomics and Outcomes Research in May 2013.

Conflict of Interest: The investigators retained full control of the study's design, conduct, and reporting.

N. Olchanski: Project funding from Medtronic, Inc.

A. Winn: Project funding from Medtronic, Inc.

J. T. Cohen: Project funding from Medtronic, Inc.

P. J. Neumann: Project funding from Medtronic, Inc.

Corresponding Author: N. Olchanski, MS; Center for the Evaluation of Value and Risk in HealthInstitute for Clinical Research and Health Policy Studies, Tufts Medical Center, Boston, MA, USA (e-mail: nolchanski@tuftsmedicalcenter.org).

\section{REFERENCES}

1. Thompson SG, Ashton HA, Gao L, Scott RA. Screening men for abdominal aortic aneurysm: 10-year mortality and cost effectiveness results from the randomised Multicentre Aneurysm Screening Study. BMJ. 2009;338:b2307.

2. Wanhainen A, Lundkvist J, Bergqvist D, Bjorck M. Cost-effectiveness of different screening strategies for abdominal aortic aneurysm. J Vasc Surg. 2005;41:741-51.

3. Giardina S, Pane B, Spinella G, et al. An economic evaluation of an abdominal aortic aneurysm screening program in Italy. J Vasc Surg. 2011;54:938-46.

4. Ehlers L, Overvad K, Sorensen J, Christensen S, Bech M, Kjolby M. Analysis of cost effectiveness of screening Danish men aged 65 for abdominal aortic aneurysm. BMJ. 2009;338:b2243.

5. Kim LG, Thompson SG, Briggs AH, Buxton MJ, Campbell HE. How cost-effective is screening for abdominal aortic aneurysms? J Med Screen. 2007;14:46-52.

6. Fieg1 C. Medicare's missed checkups: Few seniors get wellness exam. American Medical News. 2011

7. Shreibati JB, Baker LC, Hatky MA, Mell MW. Impact of the Screening Abdominal Aortic Aneurysms Very Efficiently (SAAAVE) Act on Abdominal Ultrasonography Use Among Medicare Beneficiaries. Arch Intern Med. 2012;1-7.
8. Fleming C, Whitlock EP, Beil TL, Lederle FA. Screening for abdominal aortic aneurysm: a best-evidence systematic review for the U.S. Preventive Services Task Force. Ann Intern Med. 2005; 142:203-11.

9. Scott RA, Wilson NM, Ashton HA, Kay DN. Influence of screening on the incidence of ruptured abdominal aortic aneurysm: 5-year results of a randomized controlled study. Br J Surg. 1995;82:1066-70.

10. Norman PE, Powell JT. Abdominal aortic aneurysm: the prognosis in women is worse than in men. Circulation. 2007;115:2865-9.

11. Lederle FA, Larson JC, Margolis KL, et al. Abdominal aortic aneurysm events in the women's health initiative: cohort study. BMJ. 2008;337:a1724.

12. McPhee JT, Hill JS, Eslami MH. The impact of gender on presentation, therapy, and mortality of abdominal aortic aneurysm in the United States, 2001-2004. J Vasc Surg. 2007;45:891-9.

13. Sweeting MJ, Thompson SG, Brown LC, Powell JT. Meta-analysis of individual patient data to examine factors affecting growth and rupture of small abdominal aortic aneurysms. Br J Surg. 2012;99:655-65.

14. Lederle FA. Should abdominal aortic aneurysm be managed differently in women? Scand J Surg. 2008;97:125-7.

15. Wanhainen A, Lundkvist J, Bergqvist D, Björck M. Cost-effectiveness of screening women for abdominal aortic aneurysm. J Vasc Surg. 2006;43:908-14.

16. Lederle FA, Johnson GR, Wilson SE. Abdominal aortic aneurysm in women. J Vasc Surg. 2001;34:122-6.

17. Ashton HA, Buxton MJ, Day NE, et al. The Multicentre Aneurysm Screening Study (MASS) into the effect of abdominal aortic aneurysm screening on mortality in men: a randomised controlled trial. Lancet. 2002;360:1531-9.

18. Lee TY, Korn P, Heller JA, et al. The cost-effectiveness of a "quickscreen" program for abdominal aortic aneurysms. Surgery. 2002;132:399-407.

19. Multicentre aneurysm screening study (MASS). cost effectiveness analysis of screening for abdominal aortic aneurysms based on four year results from randomised controlled trial. BMJ. 2002;325:1135.

20. Taylor DH Jr, Hasselblad V, Henley SJ, Thun MJ, Sloan FA. Benefits of smoking cessation for longevity. Am J Public Health. 2002;92:990-6.

21. Woloshin S, Schwartz LM, Welch HG. The risk of death by age, sex, and smoking status in the United States: putting health risks in context. J Natl Cancer Inst. 2008;100:845-53.

22. Lederle FA, Johnson GR, Wilson SE, et al. Prevalence and associations of abdominal aortic aneurysm detected through screening. Aneurysm Detection and Management (ADAM) Veterans Affairs Cooperative Study Group. Ann Intern Med. 1997;126:441-9.

23. Increase Utilization of Preventive Services in Medicare and Medicaid. 2012. Accessed 3-2-2014 at http://www.prevent.org/Prevention-PolicyAgenda-for-the-1 10th-Congress/Increase-Utilization-of-PreventiveServices-in-Medicare-and-Medicaid.aspx.

24. Centers for Medicare and Medicaid Services. Free Preventive Services for People in Medicare: 5.5 Million Americans on Medicare Have Used Preventive Benefits. 2011. Accessed 3-2-2014 at http://downloads.cms.gov/files/ preventionreport.pdf.

25. Mandelblatt JS, Cronin KA, Bailey S, et al. Effects of mammography screening under different screening schedules: model estimates of potential benefits and harms. Ann Intern Med. 2009;151:738-47.

26. Cohen JT, Neumann PJ, Weinstein MC. Does preventive care save money? Health economics and the presidential candidates. N Engl J Med. 2008;358:661-3.

27. Neumann PJ, Cohen JT. Cost savings and cost-effectiveness of clinical preventive care. Synth Proj Res Synth Rep. 2009.

28. Kent KC, Zwolak RM, Egorova NN, et al. Analysis of risk factors for abdominal aortic aneurysm in a cohort of more than 3 million individuals. J Vasc Surg. 2010;52:539-48. 